\title{
Influencia de la edad en el comportamiento de los carcinomas uroteliales superficiales
}

\author{
J.L. Moyano Calvo, M. Gutiérrez González, A. Pérez-Lanzac Llorca, A. Molina Carranza, \\ J.L. Álvarez-Ossorio Fernández, E. Sánchez Sánchez, J. Castiñeiras Fernández \\ Cátedra y Servicio de Urología. Hospital Universitario "Virgen Macarena" de Sevilla. \\ Fundación Joaquin Albarrán.
}

Actas Urol Esp 2005; 29 (1): 82-88

\section{RESUMEN}

INFLUENCIA DE LA EDAD EN EL COMPORTAMIENTO DE LOS CARCINOMAS UROTELIALES SUPERFICIALES Introducción: El carcinoma urotelial de vejiga es infrecuente en la población joven. Existe debate sobre la menor o igual agresividad de estos tumores en esta población. Presentamos nuestra experiencia.

Material y método: 551 tumores primarios, todos con seguimiento superior a un año salvo recidiva. Dividimos la serie en dos grupos: menores de 41 años (grupo A) y mayores de 40 años (Grupo B). Comparamos: estadio, grado, permeación linfática, multiplicidad, recidiva, progresión, sexo, T.L.E. (tiempo libre de enfermedad), volumen tumoral. Realizamos análisis estadístico uni y multivariado de las variables

Resultados: 17 tumores en menores de 41 años (31 años).

T.L.E. 54 meses en el Grupo A y 39 en el grupo B (p .028)

Recidivan el $11,8 \%$ del Grupo A y el $36 \%$ del grupo B (p .041)

Progresan el $0 \%$ del A y el 4,1\% del B (p .253)

En el Grupo A los tumores T1 (13,3\% frente a 39\%), los tumores G2 ( $0 \%$ frente a 36,7\%); sin permeación linfática ( $0 \%$ frente a 35\%) y los tumores únicos $(13,3 \%$ frente a $35,3 \%)$ recidivan significativamente menos.

En el estudio univariado apreciamos diferencias estadísticamente significativas respecto a grado (p .002), sexo (p .011)

En el multivariado son variables independientes para recidiva el estadio, "Grupo de Edad”, el realizar tto. profiláctico con BCG. Para progresión sólo el grado tiene valor pronóstico independiente.

Conclusiones:

En los menores de 41 años:

- Menor agresividad histológica $(0 \% \mathrm{G} 3)$

- La edad es un factor pronóstico independiente de recidiva pero no de progresión a pesar de un comportamiento menos "agresivo":

$-11,8 \%$ de recidiva

$-0 \%$ progresión

- T.L.E. mayor (un año más)

- Mayor incidencia en mujeres ( $41 \%$ frente $11 \%)$

- Menor recidiva de los tumores T1 (13,3\% frente a 39\%), los tumores G2 ( $0 \%$ frente a $36,7 \%)$ y los tumores únicos $(13,3 \%$ frente a $35,32 \%)$

Palabras clave: Cáncer urotelial. Factores pronósticos. Edad.

\section{ABSTRACT}

AGE'S INFLUENCE IN THE SUPERFICIAL BLADDER CANCER BEHAVIOUR

Introduction: Transitional bladder cancer is infrequent in younger people. The tumor's agresiveness in this poulation is subject of discussion.. We present our experience.

Material \& method: 551primary tumors, with at least one year of follow-up. Group A (less than 41 years old), Group B (bigger than 40 years old). We compare, stage, grade, lymphatic permeation, multiplicity, recurrence, progression, sex, T.L.E. (time free of disease), volume. We do uni and multivariate analysis.

Results: 17 tumors in youngers than 41 years $(2,1 \%)$

The recurrence rate is $11,8 \%$ in Group A and $36 \%$ in Group B (p .041)

Progresión rate: $0 \%$ in A and $4,1 \%$ in B (p .253)

In Group A, T1 tumors T1 (13,3\% vs. $39 \%)$, G2 tumors ( $0 \%$ vs. $36,7 \%)$; withoutsin lymphatic permeation ( $0 \%$ vs a $35 \%)$ and solitary tumors $(13,3 \%$ vs. $35,3 \%)$ have statistically less recurrence

In univariate analysis we apreciate statitsticaly differences in relation with grade (p .002) and sex (p .011).

In multivariate analysis, stage, "group of age" and prophylactic treatment are independent variables for recurrence, and grade for progression

Conclusions:

In youngers than 41 years old:

- Less patologic agressivity $(0 \% \mathrm{G} 3)$

- Group of age is independent prognostic variable for recurrence, but not pfor progression, despite a "less agressive behaviour": - $11,8 \%$ recurrence - $0 \%$ progression

- T.L.E. bigger (one year more)

- Higher incidence in women ( $41 \%$ vs. $11 \%)$

- Less recurrence in T1 tumors (13,3\% vs. 39\%), G2 tumors ( $0 \%$ vs $36,7 \%)$ and solitary tumors $(13,3 \%$ vs $35,32 \%)$

Key words: Bladder cancer. Prognostic factors. Age. 
$\mathrm{E}$ 1 carcinoma transicional de vejiga (CCT) aumenta su incidencia de forma directa con la edad, siendo la edad media de presentación 69 años en el varón y 81 años en la mujer ${ }^{1}$.

La incidencia por debajo de los 40 años oscila entre el $1 \%^{2}$ y el $2,4 \%^{3}$, siendo de $0,8 \%$ por debajo de $30^{4}$ y $0,4 \%$ por debajo de 20 años ${ }^{5}$.

Existen criterios dispares respecto a la agresividad de estos tumores en menores de 40 años, con autores que consideran que los tumores presentan un comportamiento menos agresivo $^{3,6,8-12,17,18}$, mientras que otros no encuentran diferencias en la agresividad en relación con la $\operatorname{edad}^{2,7,13-16}$

Presentamos los resultados de nuestra serie y hacemos un breve análisis de los resultados presentes en la literatura.

\section{MATERIAL Y MÉTODO}

551 tumores primarios con un seguimiento superior a un año salvo recidiva. Dividimos la serie en dos grupos: menores de 41 años (grupo A) y mayores de 40 años (Grupo B). Comparamos: estadio, grado, permeación linfática, multiplicidad, recidiva, progresión, sexo, T.L.E. (Tiempo Libre de Enfermedad), volumen tumoral. Realizamos análisis estadístico uni y multivariado de las variables antes mencionadas.

Se define "recidiva" como la aparición de un nuevo tumor y "progresión" como la aparición de un tumor de estadio igual o superior a T2.

El seguimiento fue cuatrimestral durante los dos primeros años, semestral en los tres siguientes y anual desde entonces. Las revisiones se realizan con ecografia abdominal, citología urinaria y cistoscopia en caso de duda diagnóstica.

El 56\% del grupo A y el 52\% del grupo realizaron tratamiento profiláctico, bien con BCG, bien con Mitomicina C (Tabla 1).

Tabla 1

Tratamiento efectuado. (p .213)

\begin{tabular}{lcc}
\hline & $\begin{array}{c}\text { Grupo A } \\
\mathrm{n}\end{array}$ & $\begin{array}{c}\text { Grupo B } \\
\mathrm{n}\end{array}$ \\
\hline No tto. & 50 & 48 \\
BCG & 48 & 42 \\
MMC & 8 & 10 \\
\hline
\end{tabular}

\section{RESULTADOS}

Tienen menos de 41 años 17 enfermos $(3,1 \%)$.

La relación de las distintas variables de estudio con los grupos de edad, así como su significación estadística se muestran en las Tablas 2 y 3. Como se puede apreciar sólo el grado (p .002) y el sexo (p .002) tienen significación estadística.

Señalar la ausencia de tumores G3 y el mayor número de tumores G1 en el Grupo A (menores de 41 años). Llama la atención el aumento de la incidencia de carcinomas en las mujeres del Grupo A, $41 \%$ frente al $11 \%$ de las mujeres del Grupo B.

El T.L.E. es significativamente mayor en el Grupo A, 54,5 meses frente a 39 meses en el Grupo B (p .028).

La tasa de recidiva en el Grupo A es significativamente menor que en el Grupo B, 11,8\% frente a 36\% (p .041). Ninguno de los enfermos del Grupo A han progresado frente al 4,1\% de los del Grupo B, tal como muestra la Tabla 4.

\section{Tabla 2}

Variables cualitativas de estudio en relación con los grupos de edad

\begin{tabular}{cccc}
\hline & $\begin{array}{c}\text { Grupo A } \\
\mathrm{n}\end{array}$ & $\begin{array}{c}\text { Grupo B } \\
\mathrm{n}\end{array}$ & $\mathrm{p}$ \\
\hline Ta & 12 & 19 & $\mathrm{n} . \mathrm{s}$. \\
$\mathrm{T} 1$ & 88 & 91 & \\
$\mathrm{G} 1$ & 65 & 29 & \\
$\mathrm{G} 2$ & 35 & 56 & .002 \\
$\mathrm{G} 3$ & 0 & 15 & \\
Lx & 29 & 31 & \\
L0 & 71 & 64 & n.s. \\
L1 & 0 & 5 & \\
Único & 88 & 72 & n.s. \\
Múltiple & 12 & 28 & \\
Hombre & 59 & 89 & .002 \\
Mujer & 41 & 11 & \\
\hline
\end{tabular}

Tabla 3

Variables cuantitativas de estudio en relación con los grupos de edad

\begin{tabular}{cccccc}
\hline & \multicolumn{2}{c}{ Grupo A } & \multicolumn{2}{c}{ Grupo B } & $\mathrm{p}$ \\
& Media & Mediana & Media & Mediana & \\
\hline T.L.E. & 54,5 & 43 & 39 & 32 & .028 \\
Volumen & 3,4 & 3 & 4,18 & 2 & n.s. \\
Edad & 34,6 & 35 & 65 & 66 & - \\
\hline
\end{tabular}


Tabla 4

Tasa de recidiva y progresión

\begin{tabular}{lccc}
\hline & $\begin{array}{c}\text { Grupo A } \\
\%\end{array}$ & $\begin{array}{c}\text { Grupo B } \\
\%\end{array}$ & $\mathrm{p}$ \\
\hline Recidiva & 11,8 & 36 & .041 \\
Progresión & 0 & 4,1 & n.s. \\
\hline
\end{tabular}

Dada la ausencia de progresión tumoral en el Grupo A, sólo analizamos la influencia de las distintas variables en la recidiva tumoral. Los resultados se muestran en la Tabla 5.

Podemos apreciar como en el Grupo A la recidiva es menor de forma estadísticamente significativa en los tumores estadio T1 (p .002); grado G2 (p .048); ausencia de permeación linfática (p .007); únicos (p .049).

Al realizar análisis multivariante para recidiva, son factores de riesgo: el estadio $\mathrm{T} 1 \mathrm{y}$ el pertenecer al grupo B, mientras que es factor protector el realizar tratamiento profiláctico bien con BCG o con MMC (Tabla 6).

Tabla 5

Variables de estudio en relación con la recidiva tumoral

\begin{tabular}{cccc}
\hline & $\begin{array}{c}\text { Grupo A } \\
\%\end{array}$ & $\begin{array}{c}\text { Grupo B } \\
\%\end{array}$ & $\mathrm{p}$ \\
\hline Ta & 0 & 22,7 & n.s. \\
T1 & 13,3 & 39 & .033 \\
G1 & 18 & 37,9 & n.s. \\
G2 & 0 & 36,7 & .048 \\
G3 & - & $29-6$ & - \\
Lx & 40 & 39 & n.s. \\
L0 & 0 & 35 & .007 \\
L1 & - & 47 & - \\
Único & 13,3 & 35,3 & .049 \\
Múltiple & 0 & 57,6 & n.s. \\
\hline
\end{tabular}

Tabla 6

Análisis multivariante para recidiva

\begin{tabular}{lccc}
\hline & $\mathrm{p}$ & Odds Ratio & Intervalo Confianza \\
\hline Estadio & $.000(\mathrm{~T} 1)$ & 3,299 & $1,903-5,720$ \\
Grado & .115 & - & - \\
Permeación linfática & .228 & - & - \\
Número & .631 & - & - \\
Volumen & .054 & - & - \\
Tto. profiláctico & .000 & &, $221-, 506$ \\
$\quad$ BCG & .000 &, 335 &, $219-, 817$ \\
$\quad$ MMC & .010 &, 423 & \\
Sexo .885 & - & - & $1,183-26,225$ \\
Grupo de edad & .030 (Grupo B) & 5,570 & \\
\hline
\end{tabular}

En el análisis multivariante para progresión tumoral, solo el grado es factor de riesgo (Tabla 7).

La curva de supervivencia libre de enfermedad para recidiva (p (log-rank) .011) se muestra en la Fig. 1 y para progresión (p (log-rank) .194) en la Fig. 2.

\section{DISCUSIÓN}

El carcinoma transicional de vejiga (CCT) aumenta su incidencia de forma directa con la edad, siendo la edad media de presentación 69 años en el varón y 81 años en la mujer ${ }^{1}$.

La incidencia por debajo de los 40 años oscila entre el $1 \%^{2}$ y el $2,4 \%^{3}$, siendo de $0,8 \%$ por debajo de $30^{4}$ y $0,4 \%$ por debajo de 20 años ${ }^{5}$.

En nuestra experiencia es de $3,1 \%$, similar a la comunicada por los autores españoles, Gasso un $2,2 \%^{16}$; Madrid un $4,1 \%{ }^{18}$.

El comportamiento de los TCC en pacientes menores de 40 años has sido desde hace muchos años objeto de discusión, tanto en su potencial agresivo como en sus características histológicas. Si realizamos una breve revisión de la literatura observamos que existen dos posturas diferentes, aquellos que consideran que los CCT en pacientes jóvenes son menos agresivos y los que consideran que no la edad no es un factor determinante en la agresividad del tumor. Aquellos que encuentran tumores de menor estadio y grado en pacientes jóvenes y los que no encuentran diferencias histopatológicas comparando con pacientes de mayor edad.

A la hora de establecer una edad de referencia la mayoría de los autores la establecen en los 40 años. Sin embargo hay autores que en menores de 40 años distinguen dos grupos, mayores o menores de 30 años, incluso a veces entre menores de treinta y veinte años ${ }^{3,8-13,17}$, con menor tasa de recidivas a menor edad.

Si bien el seguimiento de la serie es inferior a cinco años, en nuestra experiencia y de acuerdo con lo comunicado en la literatura, la mayoría de las recidivas y progresiones se producen antes de los dos años ${ }^{19-21}$, periodo ya ampliamente cubierto por 
Tabla 7

Análisis multivariante para progresión

\begin{tabular}{lccc}
\hline & $\mathrm{p}$ & Odds Ratio & Intervalo Confianza \\
\hline Estadio & .543 & - & $\mathrm{V}$ \\
Grado & .026 & & - \\
$\quad$ G2 & .342 & - & $1,298-35,211$ \\
$\quad$ G3 & .023 & 6,761 & - \\
Permeación linfática & .457 & - & - \\
Número & .503 & - & - \\
Volumen & .667 & - & - \\
Tto. profiláctico & .498 & - & - \\
Sexo .398 & - & - & - \\
Grupo de edad & .829 & - & \\
\hline
\end{tabular}

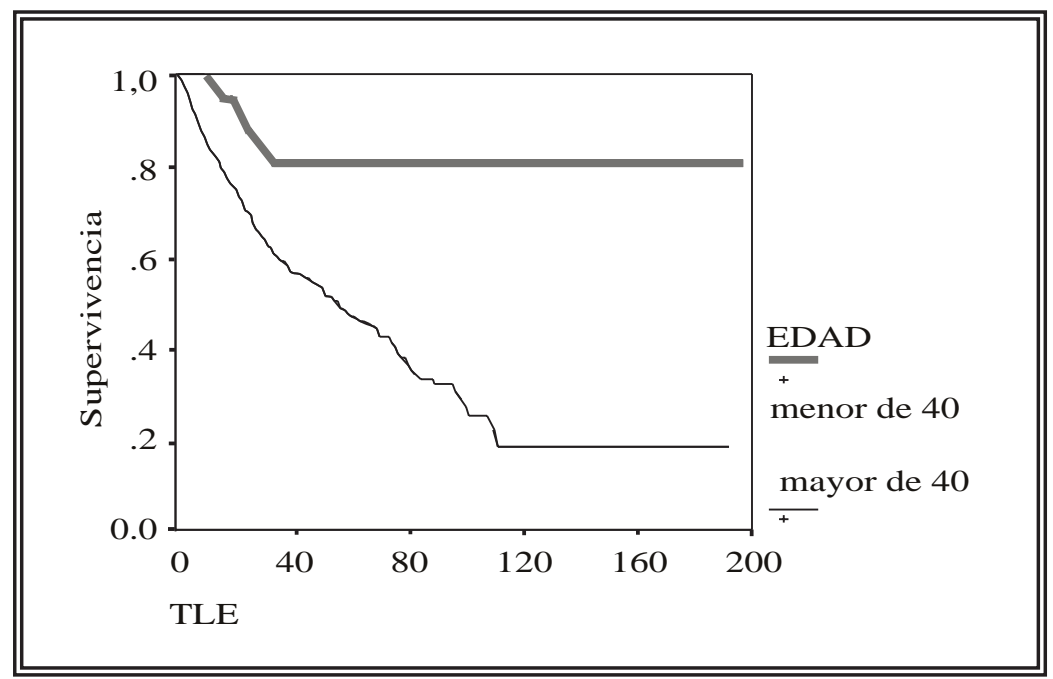

FIGURA I. TLE y Recidiva p (log-rank) .011.

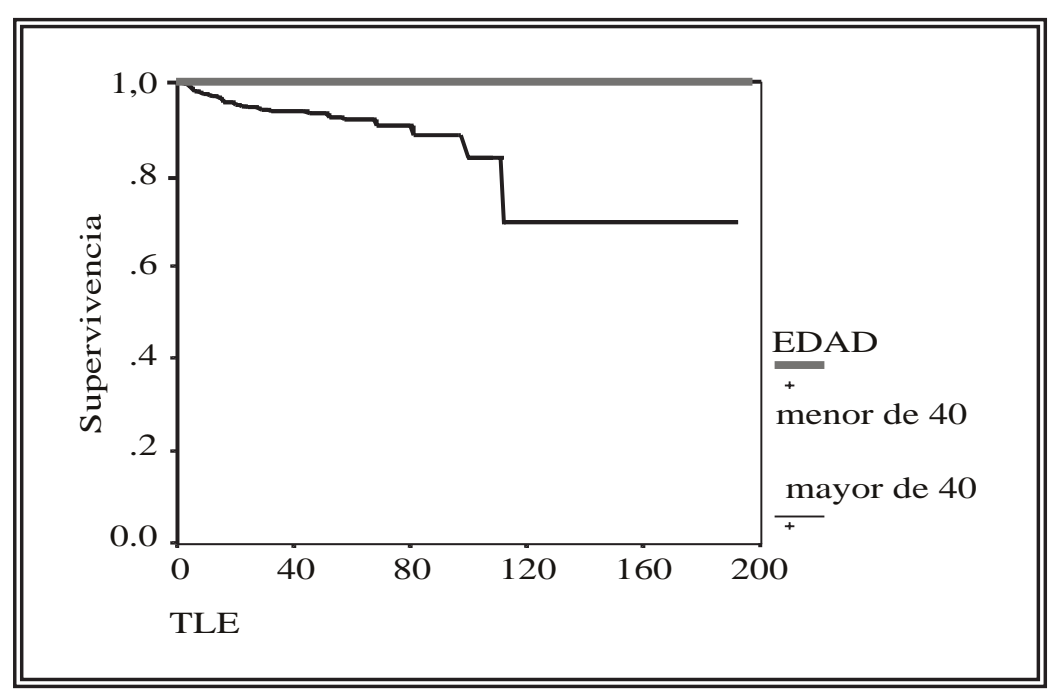

FIGURA 2. TLE y Progresión p (log-rank) .194. ambos grupos, por lo que creemos que los resultados no se modificaran sustancialmente en sucesivas revisiones.

En nuestra experiencia los pacientes con edad inferior a 41 años tienen una distribución en estadio similar pero con un grado sensiblemente menor. En el grupo A hay el doble de tumores G1 y ausencia de tumores G3 en comparación con el Grupo B. Similar resultado encuentra Yossepowitch ${ }^{14}$ cuando compara menores de 40 años con mayores de 65 tiene resultados muy similares a los nuestros, no diferencia en estadio pero sí un predominio estadísticamente significativo de los tumores de bajo grado en el grupo menor de 40 años. Si analizamos las series con pacientes menores de 40 años. Coincidimos con la mayoría de autores en este aspecto $^{6,9-11,13,16,18}$.

Un aspecto interesante, a nuestro juicio, es el aumento de la incidencia de tumores vesicales en las mujeres del Grupo A (proporción $1: 1,4$ respecto a los hombres) en comparación con las del Grupo B (proporción 1:10 respecto a los hombres). Si bien la tasa de mujeres con carcinoma vesical se sitúa entre las cifras comunicadas por la literatura, entre dos y diez veces menos frecuente que en el hombre, no deja de ser sorprendente este gran cambio de proporción. Es más, España es el país con la proporción hombremujer más alta. Es el doble que en países de Europa del Norte y EE.UU. ${ }^{22}$. Si analizamos las series con pacientes menores de 40 años, la proporción oscila entre $1: 3$ a $1: 10$, con una media de $1: 5^{2,3,6-12,14,16-18}$. Este aumento ha sido atribuido a la incorporación de la mujer a hábitos y profesiones "masculinas". Sin embargo en EEUU la incidencia de 
cáncer vesical en la mujer disminuye un $2 \%$ anualmente mientras que la del hombre sigue aumentando.

¿Tienen los tumores vesicales en menores de 40 años un comportamiento más benigno, menos agresivo?. Con técnicas inmunohistoquímicas para p53 y bcl-2 no aprecian diferencias en la inmunotinción dependiendo de la edad. Así son bcl-2 positivos el $54,1 \%$ de los más jóvenes y el 36,7\% del grupo control. Son p53 positivos el $37,5 \%$ de los más jóvenes y el 56,7\% del grupo control. Concluye que la expresión de p53 se acompaña de peor pronóstico independientemente de la edad del paciente, y aunque la expresión de bcl-2 es mayor en el grupo más joven no está asociada a recidiva o a progresión ${ }^{15}$

La mayoría de los autores coinciden en que a menor edad menor tasa de recidiva y progresión. Así:

- Kutarski ${ }^{6}$ describe un aumento de la multiplicidad recidiva y progresión conforme la edad aumenta.

- Kurz ${ }^{7}$ en 25 pacientes menores de 40 años no aprecia recidivas tumorales frente al $41 \%$ en mayores de 40 años.

- Witjes ${ }^{3}$ en 49 pacientes menores de 40 años observa que aquellos menores de 30 años tienen un comportamiento menos agresivo y un relativo mejor pronóstico. Todos los pacientes que progresaron en estadio tenían más de 30 años. En conjunto recidivaron el $30,6 \%$ de los tumores y eran infiltrantes desde el primer diagnóstico 8 tumores $(16,3 \%)$, siete de ellos en pacientes mayores de 30 años.

- Johnson y Hills ${ }^{2}$ en 22 pacientes menores de 40 años no encuentran diferencias en el comportamiento tumoral en comparación con enfermos de mayor edad. $8 / 9$ pacientes con estadio Ta-T1 están vivos en el periodo de seguimiento mientras que $11 / 13$ con estadio infiltrante han fallecido en le mismo periodo. La única diferencia que encuentran en los menores de 40 años es que tiene una menor tendencia ala multifocalidad y a la recurrencia.

- Benson ${ }^{8}$ en 12 pacientes menores de 21 años observa que todos los tumores son de bajo grado y estadio, así como el pronóstico es excelente, con una sola recidiva
- Fitzpatrick ${ }^{9}$ en cincuenta pacientes menores de cuarenta 40 años considera que los pacientes menores de treinta años tienen un comportamiento más benigno que los de mayor edad, ya que recidivaron el $8 \%$ y el $54 \%$ respectivamente

- Jemni ${ }^{10}$ en diecinueve enfermos menores de cuarenta años, también encuentra diferencias entre mayores y menores de treinta. Los menores de treinta años eran mayoritariamente superficiales (4/5), sin recidivas, progresión ni fallecimientos, mientras que en los mayores de treinta, la mitad eran superficiales $(7 / 14)$, recidivaron el $34 \%$ y fallecieron el $35 \%$.

- Chang ${ }^{11}$ en veinticinco casos menores de cuarenta años, la mayoría de ellos superficiales $(24 / 25)$ y únicos $(21 / 25)$ también aprecia diferencias en el comportamiento según lo divida en mayores o menores de treinta años, con $0 \%$ de recidivas en los menores de treintas años frente a $21 \%$ en mayores de esa edad.

- Magdar ${ }^{12}$ a veintidós pacientes menores de treinta años los divide en dos grupos, menores y mayores de 20 años, apreciando que en los menores de veinte años todos los tumores son TaG1 sin recidivas, mientras que en el grupo de los mayores de veinte años, el grado aumenta, el $40 \%$ de los enfermos presentan recurrencia y $2 / 14$ progresan a estadio infiltrante. Concluye que la recurrencia esta relacionada de manera proporcionalmente directa a la edad.

- Wan ${ }^{13}$ estudia treinta y cinco casos y los divide en dos grupos, menores y mayores de treinta años. Son infiltrantes once enfermos, ocho de ellos mayores de 30 años. Un $22 \%$ de los tumores en menores de treinta años progresan con una media de cuatro años, mientras que en los mayores de treinta años progresa el $43 \%$ con unja media de 3,5 años. Concluye diciendo que los pacientes menores de treinta años tienen una tendencia menor a presentar estadios infiltrantes y a la progresión tumoral, aunque el pronóstico depende del grado y del estadio, no de la edad de presentación.

- Yossepowitch ${ }^{14}$ compara dos grupos de diferente edad; menores de cuarenta años y mayores de sesenta y cinco años, sin apreciar diferencias significativas en el estadio, probabilidad de estar libre de recurrencia a los cinco años ni en la tasa de recidiva ni progresión. 
- Asci ${ }^{15}$ compara veinticinco pacientes menores de cuarenta años con treinta pacientes control mayores de cincuenta años. En le grupo menor de cuarenta años la tasa de recidiva fue de $16,7 \%$ y la de progresión $25 \%$, falleciendo tres pacientes $(12,5 \%)$ que tenían tumor infiltrante. En el grupo control 13,3\% recidivaron, $23,3 \%$ progresaron y $20 \%$ fallecieron por su tumor.

En España la literatura es escasa, hemos podido localizar tres artículos:

- Gasso ${ }^{16}$ en nueve casos menores de cuarenta años encuentra una histopatología más favorable con una mayor tasa de tumores superficiales, $77 \%$ y de bajo grado $44 \%$ frente al $55 \%$ y $14 \%$ respectivamente de su serie global. Sin embargo no aprecia diferencias en la tasa de recidivas en la población menor de cuarenta años, $33 \%$ frente a $42,5 \%$ del conjunto.

- Huguet ${ }^{17}$ en treinta y cuatro pacientes menores de treintas años aprecia estadio y grados bajos en el $93 \%$ y $88 \%$ de su serie, con recidiva en el $32 \%$ y progresión en el 3,5\%. Considera por tanto que estos tumores son de mejor pronóstico aunque el tratamiento debe efectuarse por el grado y estadio y no por la edad del paciente.

- Madrid ${ }^{18}$ observa bajo grado y estadio, así como baja tasa de recidiva $(12,5 \%)$ en ocho pacientes menores de cuarenta años, con similares conclusiones a las de Huguet.

En conjunto las tasa de recidiva se sitúan entre $12,5 \%$ y $32 \%$, con una media de $23 \%$, muy lejana del 50-70\% descritos en la literatura ${ }^{19-21}$.

En nuestra experiencia la tasa de recidiva es significativamente menor, $11,8 \%$ en el Grupo A frente a 36\% en el Grupo B (p .041). No hemos apreciado progresión a estadio infiltrante en el grupo A, con un seguimiento medio de 54,5 meses.

Cuando estudiamos como se comportan las variables respecto a la recidiva con relación a los grupos de edad, observamos que en el Grupo A los tumores T1 (13,3\% frente a 39\%), los tumores G2 $(0 \%$ frente a $36,7 \%)$ y los tumores únicos $(13,3 \%$ frente a $35,3 \%)$ recidivan significativamente menos. De la literatura consultada, solo dos artículos comparan grupos de distinta edad. Ni Yossepowitch (Yose) ni Asci (Asci) encuentran diferencias entre los dos grupos.
En el análisis multivariante para recidiva y progresión la variable "Grupo de Edad" tiene valor pronóstico independiente para recidiva. El pertenecer al Grupo B tiene 4,5 veces más posibilidades de recidivar. Los otros factores que tienen significación estadística (estadio, permeación linfática, multiplicidad y el realizar tratamiento profiláctico) ya han sido comunicados previamente por la literatura.

\section{CONCLUSIONES}

En los menores de 41 años apreciamos:

- Menor agresividad histológica, 0\% de tumores G3 y el doble de tumores G1 (54\% frente a $26 \%)$.

- El "Grupo de Edad" es un factor pronóstico independiente de recidiva pero no de progresión, con un riesgo cuádruple de recidiva para los mayores de 40 años.

- Un comportamiento menos "agresivo":

- $11,8 \%$ de recidiva.

- 0\% progresión.

- T.L.E. mayor (un año más).

- Mayor incidencia en mujeres (41\% frente a $11 \%)$.

- Menor recidiva de los tumores T1 (13,3\% frente a $39 \%)$, los tumores G2 ( $0 \%$ frente a $36,7 \%$ ) y los tumores únicos $(13,3 \%$ frente a $35,3 \%)$.

\section{REFERENCIAS}

1. Lynch CF, Cohen MB. Urinary system. Cancer 1995;75 (suppl):316.

2. Johnson DE, Hillis S. Carcinoma of the bladder in patients less than 40 years old. J Urol 1978;120:172-173.

3. Witjes JA, Debruyne FMJ. Bladder carcinoma in patients less than 40 yars of age. Urol Int 1989;44:81-83.

4. McCarthy JP, Gavrell GJ, LeBlanc GA. Transitional cell carcinoma of bladder patients under thitry years of age. Urology 1978;13:487-489.

5. Javadpour N, Mostofi FK. Primary eprithelial tumpos of the bladder in the first two decades of the life. J Urol 1969;101: 706-710.

6. Kutarski PW, Padwell A. Transitional cel carcinoma of the bladder in young adults. Br J Urol 1993;72:749-755.

7. Kurz KR, Pitts WR, Vaughan ED Jr. The natural history of patients less than 40 years old with bladder tumors. J Urol. 1987 mar; 137(3):395-397.

8. Benson RC Jr, Tomera KM, Kelalis PP. Transitional cell carcinoma of the bladder in children and adolescents. $J$ Urol 1983;130:54-55.

9. Fitzpatricik JM, Reda M. Bladder carcinoma in patients 40 years old or less. J Urol 1986;135:53-54.

10. Jemni M, Hassine LB, Rabaa F, El Ouakdi M, Ayed M. Les tumeurs de vessie chez le sujet de moins de 40 ans. Journal d'Urologie 1991;97:207-208. 
11. Chang SY, Ma CP. Transitional cell carcinoma of the urinary bladder in patients under 40 years of age. Br. J. Urol 1987;60:343-344.

12. Magdar I, Goldwasser B, Nativ O, Hanani Y, Jonas P. Longterm followup of patients less than 30 years old with transitional cel carcinoma of bladder. J Urol 1988;139:933-934.

13. Wan J, Grossman B. Bladder carcinoma in patients age 40 years or younger. Cancer 1989; 64:178-181.

14. Yossepowitch O, Dalbagni G. Transitional cell carcinoma of the bladder in young adults: prsentation, natural history and outcome. J Urol 2002;168:61-66.

15. Asci R, Ildis L, Sarikaya S, Buyukalpelli R, Yilmaz Af, Kandemir B. p53 and bcl-2 overexptression as asscoaites risk factors in patients 40 years old or less with transitional cell carcinma of the bladder. Urol Int 2001;67:34-40.

16. Gasso M, Pelluch A, Mejias J, Romero P, Sanchez M, Mira A. Tumores vesicales en pacientes menores de cuarenta años. Nuestra experiencia y revisión de la literatura. Arch Esp Urol 1991;44:257-258.

17. Huguet J, Errando C, Regalado R, Martínez J, Batista E, Chéchile G, Salvador J, Vicente J. Carcinoma transicional de vejiga: comportamiento en adultos jóvenes. Arch Esp Urol 1996;49:607-612.
18. Madrid FJ, Parra L, Rivas JA, Sánchez E, Monsalve M, Gómez S, García J. Carcinoma de células transicionales en pacientes menores de 40 años. Arch Esp Urol 1998;51: 991-994.

19. Morris SB, Gordon EM, Sheqarer RJ, Woodhouse CRJ. Superficial bladder cancer: for how long should a tumorfree patient have check cystoscopies. Br J urol 1995;75: 193-196.

20. Torti FM, Lum BL. The biology and treatment of superfical bladder cancer. J Clin Oncol 1984;2:505-531.

21. Van Der Meijden APM. Bladder cancer. BMJ 1998;317: 1366-1369.

22. Puente D, Malats N, Cecchini L, Tardon A, Garcia-Closas R, Serra C, Carrato A, Sala M, Boixeda R, Dosemeci M, Real FX, Kogenivas M. Gender-trelated differences in clinical and pathological characteristics and therapy of bladder cancer. Eur Urol 2003;43:53-62.

Dr. D. José Luis Moyano Calvo

C/ Bogotá 19, poertal 3, 2 $\mathrm{B}$

Sevilla 41013

(Trabajo recibido el 17 noviembre de 2003) 